\title{
Automated scoring of aortic calcification in vertebral fracture assessment images
}

DOI:

$10.1117 / 12.2512879$

\section{Document Version}

Accepted author manuscript

Link to publication record in Manchester Research Explorer

\section{Citation for published version (APA):}

Chaplin, L., \& Cootes, T. (2019). Automated scoring of aortic calcification in vertebral fracture assessment images. In K. Mori, \& H. K. Hahn (Eds.), Medical Imaging 2019: Computer-Aided Diagnosis [1095038] (Progress in Biomedical Optics and Imaging - Proceedings of SPIE; Vol. 10950). SPIE. https://doi.org/10.1117/12.2512879

\section{Published in:}

Medical Imaging 2019

\section{Citing this paper}

Please note that where the full-text provided on Manchester Research Explorer is the Author Accepted Manuscript or Proof version this may differ from the final Published version. If citing, it is advised that you check and use the publisher's definitive version.

\section{General rights}

Copyright and moral rights for the publications made accessible in the Research Explorer are retained by the authors and/or other copyright owners and it is a condition of accessing publications that users recognise and abide by the legal requirements associated with these rights.

\section{Takedown policy}

If you believe that this document breaches copyright please refer to the University of Manchester's Takedown Procedures [http://man.ac.uk/04Y6Bo] or contact uml.scholarlycommunications@manchester.ac.uk providing relevant details, so we can investigate your claim.

\section{OPEN ACCESS}




\title{
Automated scoring of aortic calcification in vertebral fracture assessment images
}

\author{
Luke Chaplin $^{\mathrm{a}}$ and Tim Cootes ${ }^{\mathrm{a}}$ \\ aDivision of Informatics, Imaging and Data Sciences, University of Manchester, Manchester, \\ UK
}

\begin{abstract}
The severity of abdominal aortic calcification (AAC) is a strong, independent predictor of cardiovascular disease (CVD). Vertebral fracture assessment (VFA) is a low radiation screening tool which can be used to incidentally measure AAC. This work compares the performance of Haar feature random forest classification with a Unet based convolutional neural network (CNN) segmentation, to automatically quantify AAC. Clinical semiquantitative scores were also generated using U-net. Scores were calculated using the relative length of labelled calcification and compared to manual scoring. The U-net outperformed the random forest, showed sensible segmentations and AAC scores, though it could not match human annotation accuracy.
\end{abstract}

Keywords: Abdominal Aortic Calcification, Cardiovascular Disease, Convolutional Neural Network, Segmentation, Image Analysis

\section{INTRODUCTION}

Cardiovascular disease (CVD) covers a range of conditions affecting the heart and blood vessels. CVD includes coronary heart disease and strokes, the biggest killers globally. ${ }^{1}$ It has been established that the severity of abdominal aortic calcification (AAC) is an independent risk factor for CVD. ${ }^{2}$ This paper describes a system for automatic estimation of AAC using vertebral fracture assessment (VFA) images.

The main driving force in the development of CVD is atherosclerosis of the arteries. Preventative treatment decisions for heart attacks and strokes are currently made using a calculated risk score. ${ }^{3}$ This risk score is calculated using a variety of lifestyle and clinical factors such as age, lipid profile and smoking history. Despite its importance in the development of CVD, measurement of atherosclerotic extent is not routinely used in these decisions. As atherosclerosis develops, calcification of the arterial wall occurs. Of particular relevance, atherosclerosis and calcification occur in the abdominal aorta. This abdominal aortic calcification (AAC) is correlated with an increased risk of heart attacks, strokes, heart failure and peripheral vascular disease, independent of other risk factors. ${ }^{2,4}$

Dual-energy x-ray absorptiometry (DXA) imaging is used primarily to measure bone mineral density in osteoporosis screening. As part of osteoporosis screening, a lateral view of the spine is acquired, to inspect for vertebral fractures. These vertebral fracture assessment (VFA) images are sensitive to signal from calcification and can be used to measure AAC. ${ }^{4,5}$ Scoring of AAC is performed using a semi-quantitative 24-point score (AAC24). ${ }^{6}$ The scoring system rates the anterior and posterior aortic wall at each vertebral level from 0-3 based on the relative length of calcified aorta. A score of 1 is given if the total length of calcifications is less than $1 / 3$, 2 if between $1 / 3$ and $2 / 3$, and 3 if more than $2 / 3$ the height of the adjacent vertebra. It is time consuming for radiologists to identify and score AAC. With a fully automated system, screening of AAC could be performed with no additional imaging or clinician time, and this valuable health data could be used to improve treatment decisions in cardiovascular disease.

There have been a number of techniques used in automatic segmentation of AAC outside of VFA images, in plain radiographs. A range of successful techniques have been applied including inpainting, ${ }^{7}$ shape guided classification and active contours, ${ }^{8}$ and appearance models with Bayesian inference. ${ }^{9}$ DXA imaging has an even lower radiation dose than plain radiography and can be used to reliably measure AAC. ${ }^{4,10}$ DXA images

Send correspondence to luke.chaplin@postgrad.manchester.ac.uk 
contain less signal from soft tissues compared to plain radiographs, but are a lower resolution and contain more noise. There has been some work on applying machine learning techniques to this problem in VFA images. ${ }^{11}$ Active appearance models were used to extract a ROI containing the aorta. Calcifications were segmented using multilevel thresholding, in order to minimise the interference of soft tissue noise. Features were then extracted using texture analysis and images classified as mild, moderate or severe using k-nearest neighbours and support vector machines. The generated classes showed very good agreement with manual scoring.

This paper continues from the excellent work so far, presenting the next stage in such a system. The performance of a random forest and a deep learning approach to segmentation are compared on a sizeable dataset of DXA VFA images. Random forests are a more traditional approach, which have yet to be applied to this problem in VFA images. Here they are compared to a deep learning architecture. Deep learning has been incredibly influential in recent years, with convolutional neural networks being especially popular in the domain of image analysis. In particular, the U-net architecture $^{12}$ and its variations have had a great deal of success in segmentation tasks. This paper demonstrates U-net can be used to improve segmentation and provide a reliable semi-quantitative measure of AAC in this important clinical scenario.

\section{METHODS}

\subsection{Data}

1600 DXA VFA images were available from the MRC National Survey of Health and Development (NSHD). ${ }^{13}$ All images were annotated on a pixel-by-pixel basis to produce an image mask of calcification, with the same resolution as each original image. Annotation was performed twice by the same reader with an interval of at least two months to gain a realistic measure of intra-rater reliability. A total of 195 images contained evidence of AAC after excluding images with an insufficient view of the spine or abdomen for AAC scoring. The NSHD dataset also provides AAC24 scores for each image. AAC24 scores ranged from 1-16, skewed towards milder calcification. These images were acquired at multiple sites using Hologic DXA systems.

Only the area of the image at the level of vertebrae L1-4 is of interest while assessing AAC. A region of interest (ROI) was therefore predicted for each VFA image containing the spine and aorta at this level. As the aorta is not visible on DXA imaging unless calcified, the location of the aorta was estimated based on vertebral landmarks using a statistical shape model (SSM). ${ }^{14}$ The SSM was trained using 30 point annotations of the spine and aorta in sagittal CT images, a modality in which the aorta is visible and its spatial relationship to the spine can be modelled. Each annotated image could then be represented as a point in a 60 dimensional space. Each of these new shapes, $\mathbf{x}_{\mathbf{i}}$, could then be represented by $\mathbf{x}_{\mathbf{i}}=\overline{\mathbf{x}}+\delta \mathbf{x}_{\mathbf{i}}$, a deviation from the mean shape. Principal component analysis was used on the covariance matrix of the deviations to produce eigenvectors which represent the principle modes of variation. A new example of the modelled shape, $\mathbf{x}^{\prime}$, could then be generated using $\mathbf{x}^{\prime}=\overline{\mathbf{x}}+\mathbf{P b}$, where $\mathbf{P}$ is the matrix of eigenvectors and $\mathbf{b}$ a vector of shape parameters. By calculating the parameters of $\mathbf{b}$ using a subset of annotations, those on the vertebrae, a new shape $\mathbf{x}^{\prime}$ was calculated to estimate the location of the aortic point annotations.

Fig. 1 shows the shape model being fitted to a VFA image. Once the aortic point annotations were estimated and a measure of error calculated, the ROI is extended by 3SD to ensure coverage of the entire aorta. For each image this ROI was warped to a consistent 128x256 grayscale image using bilinear interpolation. This warping of the image using landmarks, removes the majority of curvature of the lumbar spine and aorta, simplifying AAC score calculation. Before using spine annotations for prediction, Gaussian noise ( $\sigma=5$ pixels) was applied to their position, producing small non-linear transformations in the warped images. Applying the SSM to multiple noisy annotations allowed for the production of multiple non-linear transformations of each image, increasing the available training examples and simulating small errors in vertebral landmarks.

\subsection{Random Forest Classification}

A random forest $(\mathrm{RF})$ classifier ${ }^{15}$ was built using the 195 images, with a 5 -fold cross validation. Training data was produced by sampling 10,000 random patches from the ROI containing the aorta across the training images. Each patch was $21 \times 21$ pixels, with an equal number centred on pixels classified as positive and negative for calcification. 10 random scaling (between 0.5 and 2$)$ and rotation ( \pm 1.0 radians) transformations were applied 
A

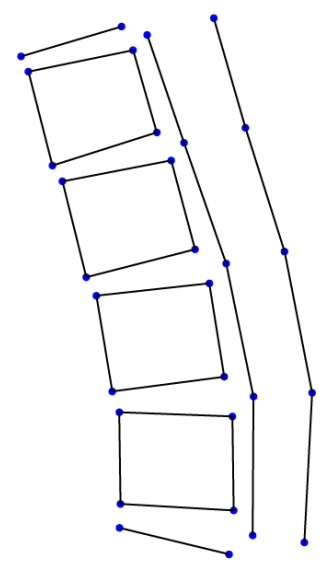

B

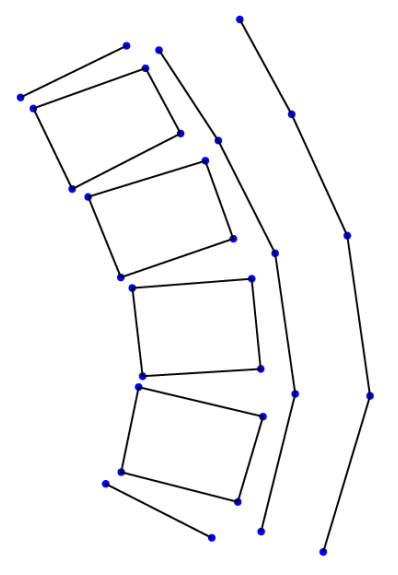

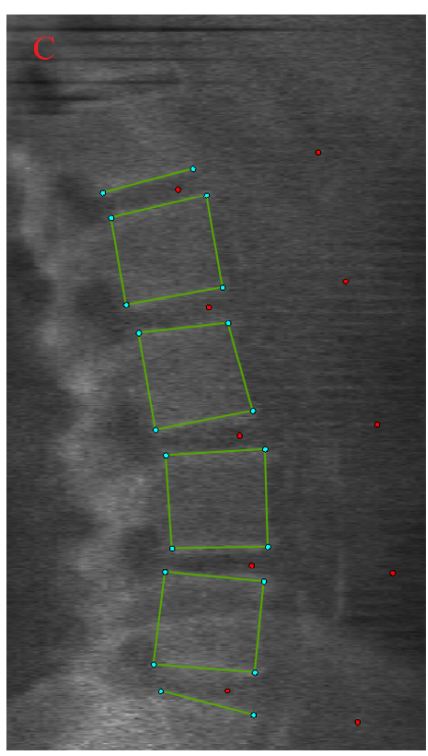

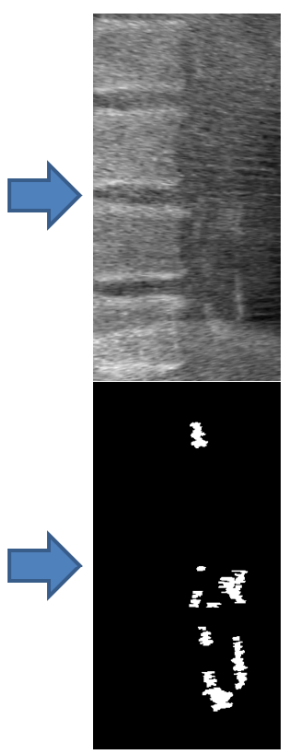

Figure 1. Fitting a shape model to a VFA image to produce a training image and label. Image A is the mean shape of the model, $\overline{\mathbf{x}}$. Image B represents the mean shape with $2 \mathrm{SD}$ added in the first principal mode of variation. Image $\mathrm{C}$ shows the shape model fitted to point annotations of the spine and the estimated aorta points. Bilinear interpolation is then used to warp the image and label into a common image size.

to each patch to increase training examples. Haar-like features ${ }^{16}$ were calculated for all patches and used to train the decision trees. 10 decision trees were trained on a bootstrapped sample of features and the class of the centre pixel. A response image was then generated for each image in the test dataset by having the RF vote on $21 \times 21$ patches in a sliding window across the whole image.

\subsection{Convolutional Neural Network Segmentation}

Segmentation of calcification was performed using a U-net architecture. ${ }^{12}$ U-net is a fully convolutional network architecture with encoding and decoding pathways which are concatenated to create skip connections. The U-net model allows semantic segmentation with an output of the same size as the input. This implementation has only a few changes from the original U-net. Batch normalisation is implemented across all convolutional layers to reduce training time. Zero padding is applied to compensate for the decrease in image size due to convolution, outputting a segmentation map of the same size as the input image with a calcification class probability at each pixel. Dropout, with probability 0.5 , is included between convolutions in the contracting path to reduce overfitting.

A 5-fold cross-validation experiment was run, producing a segmentation mask for all 195 images. For each fold $20 \%$ of images were used for validation and $20 \%$ for testing, leaving 125 images for training. For each original training image, 30 augmented images were produced using non-linear transformations from the shape model in addition to random scaling (0.95-1.05), rotation $\left(-10^{\circ}-10^{\circ}\right)$ and translation (up to $5 \%$ of image width and height in $x$ and $y$ directions respectively). Each of the 5 epochs was run on 3750 training images in batches of 4, a compromise between time and memory usage. Binary cross entropy was used as the loss score, minimised using the Adam optimiser with a learning rate of $1 \times 10^{-4}$. These network hyperparameters were determined empirically. The U-net model was built in TensorFlow using Keras, on an Nvidia Tesla K20 5GB GPU.

\subsection{Multi-Class Segmentation}

The range of possible configurations of calcification in the abdominal aorta meant that a semi-quantitative score could not be reliably generated without being able to automatically distinguish calcification on the anterior and posterior walls. To achieve this, the training images were annotated with a separate class for anterior and posterior calcification. The same U-net architecture was then adapted to take a multi-class input and produce a 
probability for each class at each pixel in the final sigmoid layer. The multi-class segmentation was also trained for 5 epochs with a learning rate of $1 \times 10^{-4}$, on 3750 training images in batches of 4 .

\subsection{AAC24 Scoring}

In order to produce AAC24 scores the midline of the aorta and intervertebral spaces was estimated. During the bilinear interpolation warp to create the dataset, the positions of the intervertebral spaces were transformed to consistent locations (64 pixels apart) within the image. The removal of the majority of aortic concavity or convexity during the production of the dataset allowed the midline of the aorta to be approximated with a vertical line. This line was estimated by minimising a cost function that rewards having anterior class pixels on the anterior side of the line, punishes their presence on the posterior side, and vice versa. This cost $C$ was calculated using a sigmoid function as shown:

$$
C(\mathbf{x}, l)=\sum_{j=1}^{j_{n}} \frac{1}{1+e^{-c\left(x_{j}-l\right)}}
$$

Where $x_{j}$ is the position of anterior or posterior pixel $j$ on the $x$-axis, $l$ is the position of the candidate vertical line on the $\mathrm{x}$ axis, and $c_{j}$ is the class of pixel $\mathrm{j}$ ( 1 for anterior and -1 for posterior).

Once the midline of the aorta had been estimated for each image, the number of classified pixels on the correct side of the midline in each row of the image was totalled. The proportion of rows in each vertebral length with at least one positive pixel was then calculated. AAC scores were then produced according to equations (2).

$$
A A C 24=\sum_{v=1}^{4}\left\lceil\left(3 \frac{a_{v}}{h_{v}}\right)\right\rceil+\left\lceil\left(3 \frac{p_{v}}{h_{v}}\right)\right\rceil
$$

Where $a_{v}$ and $p_{v}$ are the number of rows of vertebra $v$ containing correctly assigned anterior and posterior pixels respectively. $h_{v}$ is the height of vertebra $v$ in pixels, which in all instances was 64 pixels. These scores were calculated for all predicted images from the multi-class U-net and all training annotations to compare the segmentation performance.

\section{RESULTS}

\subsection{Random Forest and U-net Segmentation}

All 195 images were used for testing as part of a 5-fold cross-validation experiment, creating predicted label images from both RF classification and U-net segmentation. Fig. 2 shows a comparison of the manual training annotations and label images produced by the U-net and RF overlaid on the test image. Qualitatively the RF predicted labels are more noisy, with false positives distributed throughout the image. Morphology techniques can be used to address the large number of false positives, but dilation and erosion also heavily impact the true positive labels. In general, the U-net produces convincing annotations of calcifications, as seen in image A of Fig. 2. Image B demonstrates an extreme example of the U-net's tendency to underestimate the volume of calcifications.

Table 1. Comparison of segmentation techniques using the mean Dice similarity coefficient (DSC) as a performance measure, standard deviation across the dataset is also included. The Jaccard similarity coefficient (JSC) and accuracy (ACC) were also calculated for comparison with previous work.

\begin{tabular}{|c|c|c|c|}
\hline Method & DSC (SD) & JSC (SD) & ACC (SD) \\
\hline Random Forest & $0.373(0.149)$ & $0.240(0.112)$ & $98.6 \%(0.843 \%)$ \\
U-net & $0.569(0.189)$ & $0.420(0.170)$ & $99.1 \%(0.725 \%)$ \\
\hline
\end{tabular}



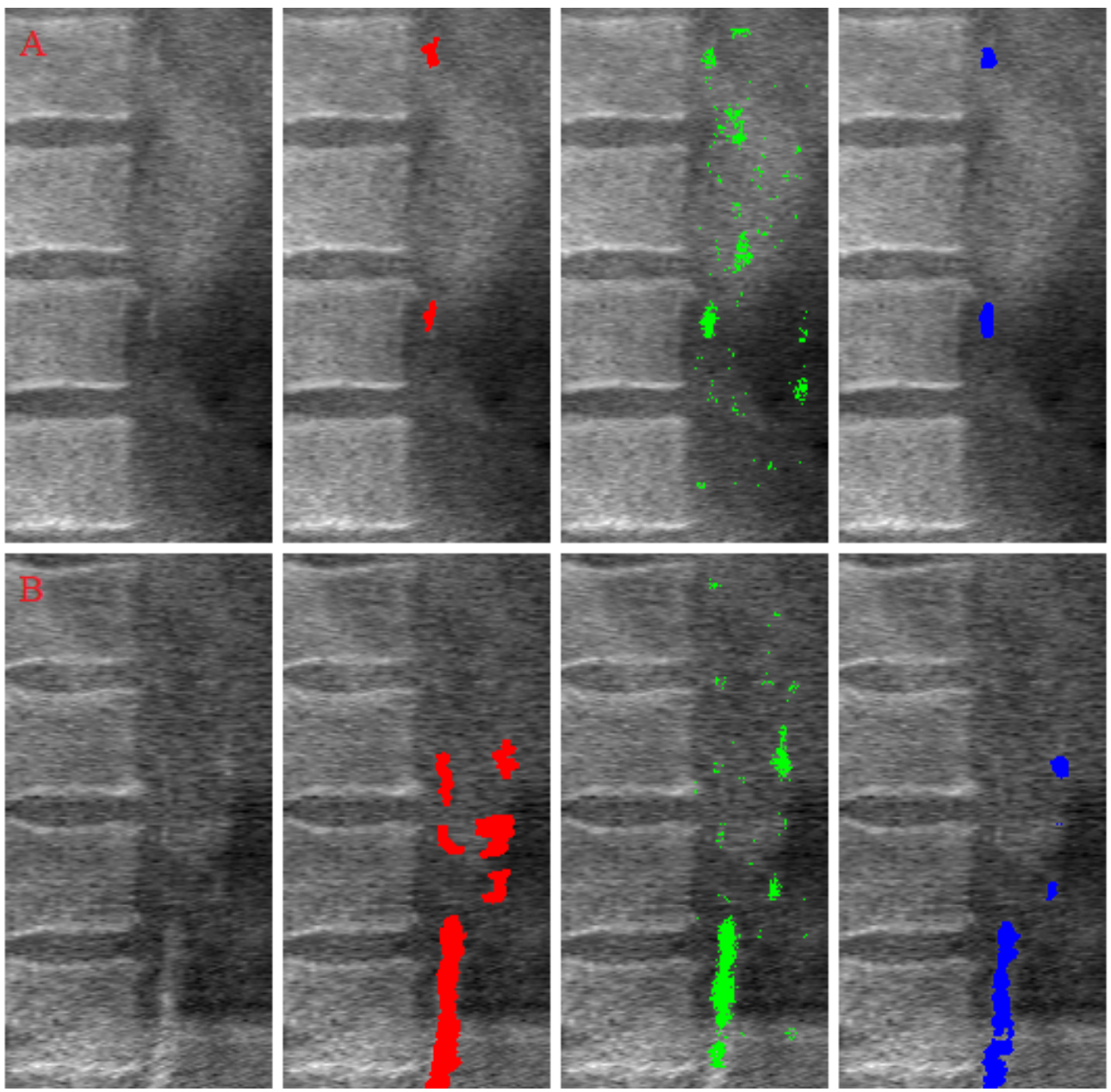

Figure 2. A comparison of the labels produced by the segmentation techniques. From left to right the images are: the warped ROI used to test, the manual annotation used for training, the label output of the RF, and the output of the U-net segmentation is rightmost. 

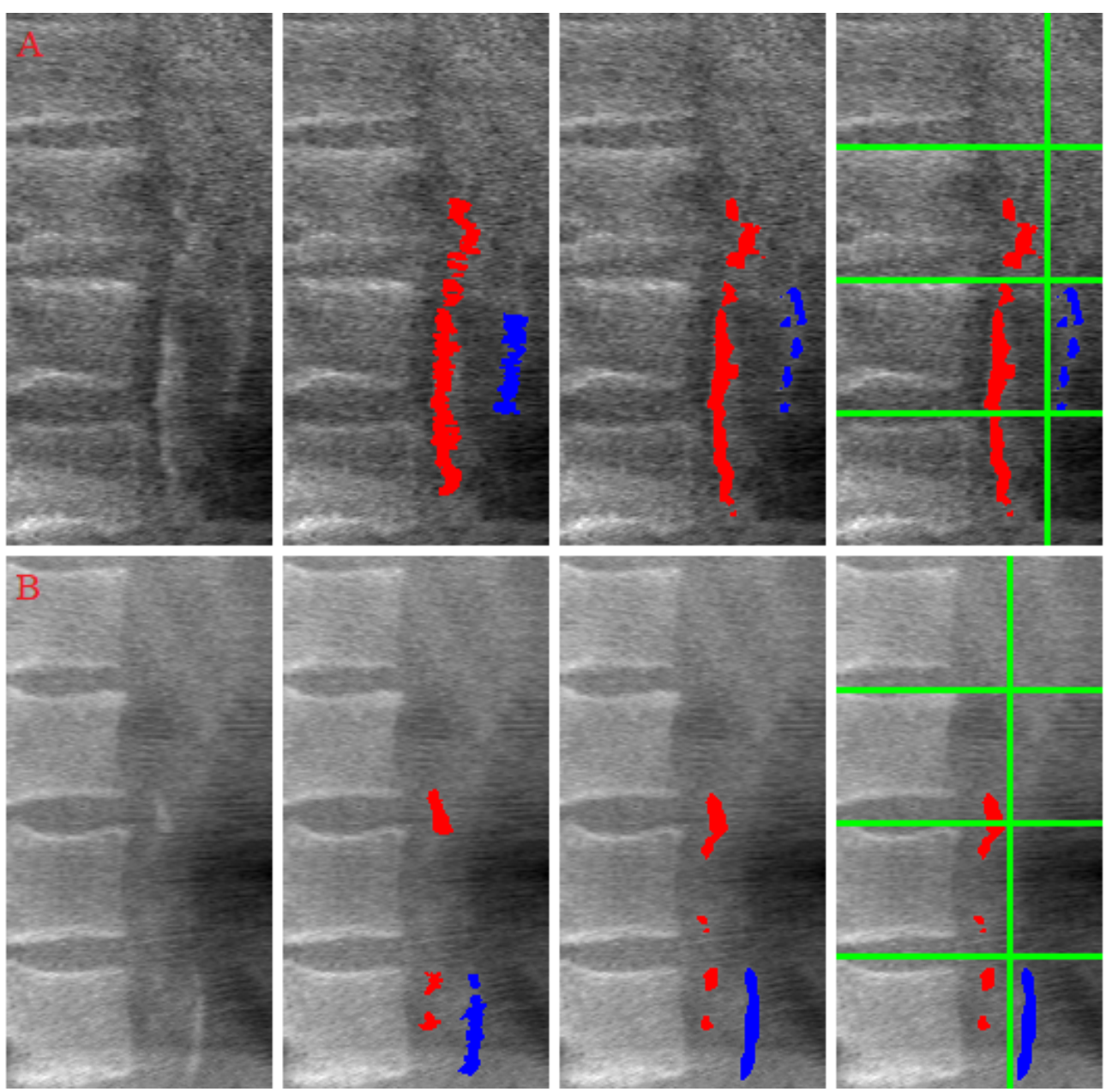

Figure 3. Examples of the multi-class U-net segmentation performance, compared to manual annotation and the binary U-net. The anterior class is shown in blue, with posterior in red. From left to right the images are: the warped ROI used to test, the manual annotation used for training, the output of the multi-class U-net, the aortic midline fitting to calculate a AAC24 score.

To quantify performance, the Dice similarity coefficient (DSC) was calculated between the manual annotations and thresholded predicted label images. Table 1 summarises the performance of each technique. Accuracy and the Jaccard similarity coefficient (JSC) were also calculated. Accuracy is not a particularly useful metric in this problem, as the overwhelmingly large number of true negatives can cause the values to be misleading, but is included for comparison to previous approaches. As an overlap metric, DSC is sensitive to misaligning of small patches, so has a relatively high variance over the dataset. A second annotation of the dataset was also performed, to gain a measure of intra-rater reliability, the DSC for these two annotations was 0.869 . The performance of the U-net segmentation was a significant improvement over the RF but could not approach the accuracy of the repeated annotation.

\subsection{Multi-Class Segmentation and Scoring}

A second 5-fold cross-validation experiment was undertaken, training a multi-class U-net on two-class annotations of anterior and posterior wall calcification. Fig. 3 shows some examples of the two-class annotation and the predicted label image from the U-net segmentation. The performance for the multi-class U-net is qualitatively similar to the binary U-net. DSC was lower at 0.525, owing mostly to increased uncertainty of the anterior class. Unlike the posterior wall, which is adjacent to the spine, the location of the anterior wall can vary far more with 

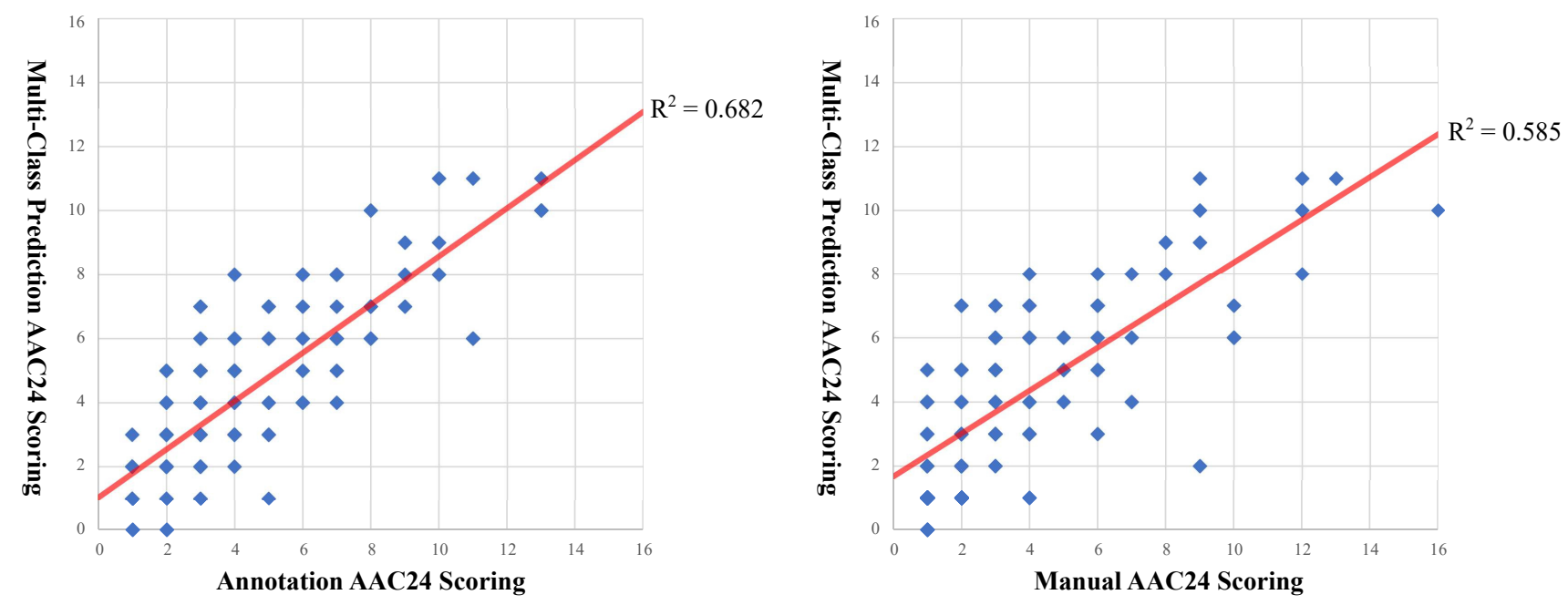

Figure 4. Comparison of the AAC24 scores produced by aortic midline estimation. The left chart compares the application of the midline algorithm to the manual annotations of calcification and those produced by the multi-class U-net. The right chart shows the correlation between manual AAC24 scores from the original VFA images, and the predicted scores based on U-net segmentation.

anatomy. In some predictions this lead to the misclassification of posterior calcification as anterior, impacting overlap measures.

Each label produced by the multi-class U-net was used to produce an AAC24 score based on the maximum proportion of each vertebral level, anterior and posterior, that was segmented as calcification. The rightmost images of Fig. 3 show the estimation of the midline of the aorta and the vertebral separations. Image A demonstrates a common scenario in which a prediction underestimating the size of calcification leads to a lower DSC but does not heavily impact the final AAC24 score. Image B is included to demonstrate situations in which small false positives can impact overall scoring. Fig. 4 compares the AAC24 scores calculated from the training annotations with those from the U-net segmentations, with a $R^{2}$ coefficient of 0.682 . Restricting the trend line to pass through the origin, which is expected for an identical scoring method applied to both datasets, reduces the $R^{2}$ coefficient to 0.630 . Fig. 4 also demonstrates the correlation between radiologist produced AAC24 scores from the NSHD dataset and those generated automatically from the U-net segmentation, with a $R^{2}$ coefficient of 0.585 .

\section{DISCUSSION}

An automated method for the segmentation of abdominal aortic calcification in vertebral fracture assessment images has been proposed. These segmentations were then used to produce a semi-quantitative clinical score, AAC24. Segmentations from both the RF and U-net appeared to identify the majority of calcifications in the dataset. The overlap metrics for U-net were a significant improvement over RF. The RF performance was heavily impacted by a large number of false positive areas. This is likely a result of the RF having only local image features to use for classification combined with the large amount of noise and soft tissue signal. It is possible that the random forest is sensitive to early calcification that a human reader cannot detect, but this is difficult to demonstrate without access to radiograph or $\mathrm{CT}$ images of the same individual. Despite the superior performance of U-net, RF approaches should not be discounted. As they can still identify the majority of calcification without the need for as much training time or GPU hardware. RF may also transfer better to other datasets, as decisions are made using local intensity features.

U-net segmentation also compared favourably to previous work segmenting AAC in radiographs. The performance of the shape model and particle filter techniques from DeBrujne ${ }^{17,18}$ use accuracy as the performance metric. Although the value of accuracy can be misleading without knowing the ratio of true and negative pixels, 
the U-net's higher accuracy is promising. Lauze and DeBrujne ${ }^{8}$ went on to combine the techniques of pixel classification, active shape models, particle filtering and inpainting for segmentation. The JSC of this approach was a very similar 0.421. Petersen et al. ${ }^{9}$ used appearance models, random forests and Bayesian inference to segment the aorta and calcifications and then went on to examine how the estimated measurements effect CVD risk. The U-net performed significantly better than the segmentation performance, though this is secondary to the aim of improving CVD prediction with an elegant survival analysis. Elmasri et al. ${ }^{11}$ have tackled the challenge of automated AAC segmentation in VFA images. Using active appearance models and thresholding to generate features used by k-nearest neighbours and support vector machines to classify AAC into three classes (mild, moderate and severe). The performance of the technique is impressive, though prediction performance for AAC24 scores is not addressed for comparison. Many of the techniques from these papers used for CVD prediction and $\mathrm{AAC} 24$ scoring could be combined with the segmentation performance of this paper.

The correlation between automatically generated scores and manual scores is very encouraging. With a $R^{2}$ coefficient of 0.585 , this technique surpassed the 0.49 achieved by Petersen et al. ${ }^{9}$ in lateral radiographs. This performance is of note, given that it was achieved on VFA images with lower resolution and increased noise. The midline scoring system performed well but likely suffers from being too strict in its definitions. When a radiologist is manually assessing an image for AAC, they do not measure the exact heights of calcifications. Situations in which a single area of calcification bridges two vertebral sections are less likely to contribute to both scores when manually assessed. AAC24 scoring is designed to enable quick and consistent assessment, which requires the system be more flexible to improve correlation.

This paper has demonstrated deep learning and random forest approaches to automated segmentation of AAC in VFA images. As well as the production of clinical semi-quantitative scores using a multi-class Unet segmentation. Visual inspection of U-net segmentation indicates that the majority of calcification is being identified, and quantitative performance was superior to an RF approach. There has been comparable agreement with previous work on plain radiographs, which have higher resolution and reduced noise. This is a promising continuation of previous work in an area with considerable clinical potential. U-net performance should only improve with additional data, though the subjective nature of annotating individual pixels containing calcification will limit the upper bound on overlap scores. Additional data, with multiple expert annotations, should improve segmentation and provide a target for performance. Petersen et al. ${ }^{9}$ provided a measure of inter-rater reliability for annotation at $J S C=0.51$ which is not too dissimilar from the U-net 0.420. Future work will focus on improving system accuracy with more varied training data, production of continuous measures of AAC and investigation into how these measures can inform CVD risk scores.

\section{ACKNOWLEDGMENTS}

We wish to thank the NSHD study members for their continued participation in the study. We also thank the NSHD study staff and the UK Medical Research Council for creating, running and facilitating access to the study. We would like to acknowledge the important role of the late Professor Judith Adams in this project, and thank her for the support she gave. We thank Drs Paul Bromiley, Michael Machin and Xinghui Dong for their help facilitating data access and collection. We also thank Dr Adrian Davison for his support and advice. Author L. Chaplin is funded by the UK Medical Research Council (Grant Number: MR/N013751/11).

\section{REFERENCES}

[1] World Health Organisation, "Deaths by Cause, Age, Sex, by Country and by Region, 2000-2016." Global Health Estimates 2016 http://www.who.int/healthinfo/global\{_\}burden\{_\}disease/estimates/en/ index1.html. (Accessed 13 March 2018).

[2] Bastos Gonçalves, F., Voûte, M. T., Hoeks, S. E., Chonchol, M. B., Boersma, E. E., Stolker, R. J., and Verhagen, H. J. M., "Calcification of the abdominal aorta as an independent predictor of cardiovascular events: a meta-analysis.," Heart (British Cardiac Society) 98(13), 988-994 (2012).

[3] Collins, G. S. and Altman, D. G., "Predicting the 10 year risk of cardiovascular disease in the United Kingdom: Independent and external validation of an updated version of QRISK2," BMJ (Online) $\mathbf{3 4 5}(7867)$, $1-12(2012)$. 
[4] Schousboe, J. T., Taylor, B. C., Kiel, D. P., Ensrud, K. E., Wilson, K. E., and McCloskey, E. V., "Abdominal Aortic Calcification Detected on Lateral Spine Images From a Bone Densitometer Predicts Incident Myocardial Infarction or Stroke in Older Women," Journal of Bone and Mineral Research 23(3), 409-416 (2007).

[5] Bazzocchi, A., Ciccarese, F., Diano, D., Spinnato, P., Albisinni, U., Rossi, C., and Guglielmi, G., "DualEnergy X-Ray Absorptiometry in the Evaluation of Abdominal Aortic Calcifications," Journal of Clinical Densitometry 15(2), 198-204 (2012).

[6] Kauppila, L. I., Polak, J. F., Cupples, L. A., Hannan, M. T., Kiel, D. P., and Wilson, P. W. F., "New indices to classify location, severity and progression of calcific lesions in the abdominal aorta: A 25-year follow-up study," Atherosclerosis 132(2), 245-250 (1997).

[7] Conrad-Hansen, L. a., de Bruijne, M., Lauze, F., Tankó, L. B., Pettersen, P. C., He, Q., Chen, J., Christiansen, C., and Nielsen, M., "Quantifying calcification in the lumbar aorta on X-ray images.," Medical image computing and computer-assisted intervention : MICCAI ... International Conference on Medical Image Computing and Computer-Assisted Intervention 10(Pt 2), 352-9 (2007).

[8] Lauze, F. and de Bruijne, M., "Toward automated detection and segmentation of aortic calcifications from radiographs," in [Proc. SPIE 6512, Medical Imaging 2007: Image Processing], Pluim, J. P. W. and Reinhardt, J. M., eds., 651239-7 (2007).

[9] Petersen, K., Ganz, M., Mysling, P., Nielsen, M., Lillemark, L., Crimi, A., and Brandt, S. S., "A Bayesian Framework for Automated Cardiovascular Risk Scoring on Standard Lumbar Radiographs," IEEE Transactions on Medical Imaging 31, 663-676 (mar 2012).

[10] Setiawati, R., Di Chio, F., Rahardjo, P., Nasuto, M., Dimpudus, F. J., and Guglielmi, G., "Quantitative Assessment of Abdominal Aortic Calcifications Using Lateral Lumbar Radiograph, Dual-Energy X-ray Absorptiometry, and Quantitative Computed Tomography of the Spine," Journal of Clinical Densitometry 19(2), 242-249 (2016).

[11] Elmasri, K., Hicks, Y., Yang, X., Sun, X., Pettit, R., and Evans, W., "Automatic Detection and Quantification of Abdominal Aortic Calcification in Dual Energy X-ray Absorptiometry," Procedia Computer Science 96, 1011-1021 (2016).

[12] Ronneberger, O., Fischer, P., and Brox, T., "U-Net: Convolutional Networks for Biomedical Image Segmentation," in [Medical Image Computing and Computer-Assisted Intervention MICCAI 2015], Springer, Cham (2015).

[13] Kuh, D., Adams, J., Cooper, C., and Prentice, A., "MRC NSHD 2006 - 2012 Muscle and Bone Specialist Data," (2016).

[14] Cootes, T., Taylor, C., Cooper, D., and Graham, J., "Active Shape Models-Their Training and Application," Computer Vision and Image Understanding 61(1), 38-59 (1995).

[15] Breiman, L., "Bagging predictors," Machine Learning 24(2), 123-140 (1996).

[16] Viola, P. and Jones, M., "Rapid object detection using a boosted cascade of simple features," in [Proceedings of the 2001 IEEE Computer Society Conference on Computer Vision and Pattern Recognition. CVPR 2001], 1, 511-518, IEEE Comput. Soc (2001).

[17] de Bruijne, M., "Shape Particle Guided Tissue Classification," in [2006 Conference on Computer Vision and Pattern Recognition Workshop (CVPRW'06)], 2006, 64-64, IEEE (2006).

[18] de Bruijne, M., "A Pattern Classification Approach to Aorta Calcium Scoring in Radiographs," Computer Vision for Biomedical Image Applications 3765, 170-177 (2005). 